\title{
A Fast Newton-Shamanskii Iteration for a Matrix Equation Arising from M/G/1-Type Markov Chains
}

\author{
Pei-Chang Guo \\ School of Science, China University of Geosciences, Beijing 100083, China \\ Correspondence should be addressed to Pei-Chang Guo; peichang@cugb.edu.cn
}

Received 16 June 2017; Revised 18 September 2017; Accepted 28 September 2017; Published 19 October 2017

Academic Editor: Nunzio Salerno

Copyright (C) 2017 Pei-Chang Guo. This is an open access article distributed under the Creative Commons Attribution License, which permits unrestricted use, distribution, and reproduction in any medium, provided the original work is properly cited.

For the nonlinear matrix equations arising in the analysis of M/G/1-type and GI/M/1-type Markov chains, the minimal nonnegative solution $G$ or $R$ can be found by Newton-like methods. We prove monotone convergence results for the Newton-Shamanskii iteration for this class of equations. Starting with zero initial guess or some other suitable initial guess, the Newton-Shamanskii iteration provides a monotonically increasing sequence of nonnegative matrices converging to the minimal nonnegative solution. A Schur decomposition method is used to accelerate the Newton-Shamanskii iteration. Numerical examples illustrate the effectiveness of the Newton-Shamanskii iteration.

\section{Introduction}

Some necessary notation for this article is as follows. For any matrix $B=\left[b_{i j}\right] \in \mathbb{R}^{n \times n}, B \geq 0(B>0)$ if $b_{i j} \geq 0\left(b_{i j}>0\right)$ for all $i, j$; for any matrices $A, B \in \mathbb{R}^{n \times n}, A \geq B(A>B)$ if $a_{i j} \geq b_{i j}\left(a_{i j}>b_{i j}\right)$ for all $i$, $j$; the vector with all entries equal to one is denoted by $e$; that is, $e=(1,1, \ldots, 1)^{T}$; and the identity matrix is denoted by $I$. An M/G/1-type Markov Chain (MC) is defined by a transition probability matrix of the form

$$
P=\left[\begin{array}{cccccc}
B_{0} & B_{1} & B_{2} & B_{3} & \cdots \\
C & A_{1} & A_{2} & A_{3} & \cdots \\
& A_{0} & A_{1} & A_{2} & \ddots \\
& & A_{0} & A_{1} & \ddots \\
& & & & \\
0 & & & \ddots & \ddots
\end{array}\right],
$$

while the transition probability matrix of a GI/M/1-type MC is as follows:

$$
P=\left[\begin{array}{ccccc}
B_{0} & C & & & 0 \\
B_{1} & A_{1} & A_{0} & & \\
B_{2} & A_{2} & A_{1} & A_{0} & \\
B_{3} & A_{3} & A_{2} & A_{1} & \ddots \\
\vdots & \vdots & \ddots & \ddots & \ddots
\end{array}\right],
$$

where $B_{0} \in \mathbb{R}^{m_{0} \times m_{0}}$ and $A_{1} \in \mathbb{R}^{m \times m}$, respectively. $N$ is the smallest index $i$ such that $A_{i}$, for $i>N$, is (numerically) zero. The steady-state probability vector of an M/G/1-type MC, if it exists, can be expressed in terms of a matrix $G$ that is the element-wise minimal nonnegative solution to the nonlinear matrix equation [1]

$$
G=\sum_{i=0}^{N} A_{i} G^{i}
$$

Similarly, for the GI/M/1-type MC, a matrix $R$ is of practical interest, which is the element-wise minimal nonnegative solution to the nonlinear matrix equation [2]

$$
R=\sum_{i=0}^{N} R^{i} A_{i}
$$


For a large number of stochastic models, most steady-state and certain transient characteristics of the process can be expressed in terms of the minimal nonnegative solution described above. This problem arises in the analysis of queues with phase type service times, as well as in queues that can be represented as quasi-birth-death processes [3]. It is known that any M/G/1-type MC can be transformed into a GI/M/1type MC and vice versa through either the Ramaswami [4] or Bright [5] dual, and the $G(R)$ matrix can be obtained directly in terms of the $R(G)$ matrix of the dual chain. The drift of the chain is defined by

$$
\rho=p^{T} \beta
$$

where $p$ is the stationary probability vector of the irreducible stochastic matrix $A=\sum_{i=0}^{N} A_{i}$ and $\beta=\sum_{i=1}^{N} i A_{i} e$. The MC is positive and recurrent if $\rho<1$, null and recurrent if $\rho=$ 1 , and transient if $\rho>1$; and throughout this article it is assumed that $\rho \neq 1$.

Available algorithms for finding the minimal nonnegative solution to (3) include functional iterations [1], pointwise cyclic reduction (CR) [6], the invariant subspace (IS) approach [7], the Ramaswami reduction (RR) [8], and the Newton iteration (NI) [3, 9-11]. For a detailed comparison of these algorithms, we refer the readers to [11] and the references therein. In [11], Newton's iteration is revisited and accelerated. From numerical experience, the fast Newton's iteration in [11] is a very competitive algorithm.

In this paper, we consider the Newton-Shamanskii iteration for (3). It is shown that, starting with a suitable initial guess, the sequence generated by the Newton-Shamanskii iteration is monotonically increasing and converges to the minimal nonnegative solution of (3). Similar to Newton's iteration, equation involved in the Newton-Shamanskii iteration step is a linear equation of the form $\sum_{j=0}^{N-1} B_{j} X C^{j}=E$, which can be solved fast by a Schur decomposition method. The Newton-Shamanskii iteration differs from Newton's iteration as the Fréchet derivative is not updated at each iteration; therefore the special coefficient matrix structure form can be reused.

The paper is organized as follows. The Newton-Shamanskii iteration and its accelerated iterative procedure using a Schur decomposition method are given in Section 2. Then M/G/1-type MCs with low-rank downward transitions and low-rank local and upward transitions are considered in Sections 3 and 4, respectively. Numerical results in Section 6 show that the fast Newton-Shamanskii iteration can be more efficient than the fast Newton's iteration proposed in [11]. Final conclusions are presented in Section 6.

\section{Newton-Shamanskii Iteration}

In this section, we present the Newton-Shamanskii iteration for (3). First we rewrite (3) as

$$
\mathscr{G}(X)=\sum_{v=0}^{N} A_{v} X^{v}-X=0
$$

The function $\mathscr{G}$ is a mapping from $\mathbb{R}^{m \times m}$ into itself and the Fréchet derivative of $\mathscr{G}$ at $X$ is a linear map $\mathscr{G}_{X}^{\prime}: \mathbb{R}^{m \times m} \rightarrow$ $\mathbb{R}^{m \times m}$ given by

$$
\mathscr{G}_{X}^{\prime}(Z)=\sum_{\nu=1}^{N} \sum_{j=0}^{v-1} A_{v} X^{j} Z X^{v-1-j}-Z .
$$

The second derivative at $X, \mathscr{G}_{X}^{\prime \prime}: \mathbb{R}^{m \times m} \rightarrow \mathbb{R}^{m \times m}$, is given by

$$
\begin{aligned}
\mathscr{G}_{X}^{\prime \prime}\left(Z_{1}, Z_{2}\right) \\
=\sum_{v=2}^{N} \sum_{j=0}^{v-1} A_{v}\left(\sum_{i=0}^{j-1} X^{i} Z_{2} X^{j-1-i}\right) Z_{1} X^{v-1-j} \\
\quad+\sum_{v=2}^{N} \sum_{j=0}^{v-2} A_{v} X^{j} Z_{1}\left(\sum_{i=0}^{v-2-j} X^{i} Z_{2} X^{v-2-j-i}\right) .
\end{aligned}
$$

For a given initial guess $G_{0,0}$, the Newton-Shamanskii iteration for the solution of $\mathscr{G}(x)=0$ is as follows.

For $k=0,1, \ldots$,

$$
\begin{aligned}
\mathscr{G}_{G_{k, 0}}^{\prime} X_{k, s-1} & =-\mathscr{G}\left(G_{k, s-1}\right), \\
G_{k, s} & =G_{k, s-1}+X_{k, s-1}, \\
\qquad s=1,2, \ldots, n_{k}, & \\
G_{k+1} & =G_{k+1,0}=G_{k, n_{k}} .
\end{aligned}
$$

$X_{k, s-1}$ is the solution to

$$
\begin{gathered}
X_{k, s-1}-\sum_{v=1}^{N} \sum_{j=0}^{v-1} A_{v} G_{k}^{j} X_{k, s-1} G_{k}^{v-1-j} \\
=\sum_{v=0}^{N} A_{v} G_{k, s-1}^{v}-G_{k, s-1},
\end{gathered}
$$

which, after rearranging the terms, can be rewritten as

$$
\begin{gathered}
X_{k, s-1}-\sum_{j=0}^{N-1} \sum_{v=j+1}^{N} A_{v} G_{k}^{v-1-j} X_{k, s-1} G_{k}^{j} \\
=\sum_{v=0}^{N} A_{v} G_{k, s-1}^{v}-G_{k, s-1} .
\end{gathered}
$$

Following the notation of [11], we define $S_{k, i}=\sum_{j=i}^{N} A_{j} G_{k}^{j-i}$; then the above equation is

$$
\begin{aligned}
& \left(S_{k, 1}-I\right) X_{k, s-1}+\sum_{j=1}^{N-1} S_{k, j+1} X_{k, s-1} G_{k}^{j} \\
& =G_{k, s-1}-\sum_{v=0}^{N} A_{v} G_{k, s-1}^{v},
\end{aligned}
$$

which is a linear equation of the same form $\sum_{j=0}^{N-1} B_{j} X C^{j}=E$ as the Newton's iteration step. It can be solved fast by applying 
a Schur decomposition on the matrix $C$, which is the $m \times m$ matrix $G_{k}$ here, and then solving $m$ linear systems with $m$ unknowns and equations. For the detailed description for solving $\sum_{j=0}^{N-1} B_{j} X C^{j}=E$, we refer the reader to $[11,12]$. We stress that, for Newton-Shamanskii iteration, the coefficient matrices are updated once after every $n_{k}$ iteration step and the special coefficient structure can be reused, so the cost per iteration step is reduced significantly.

\section{The Case of Low-Rank Downward Transitions}

When the matrix $A_{0}$ is of rank $r$, meaning that it can be decomposed as $A_{0}=\widehat{A}_{0} \Gamma$ with $\widehat{A}_{0} \in \mathbb{R}^{m \times r}$ and $\Gamma \in \mathbb{R}^{r \times m}$, we refer to the $\mathrm{MC}$ as having low-rank downward transitions. If Newton-Shamanskii iteration is applied to this case, all the matrices $X_{k, s-1}$ can be written as $\widehat{X}_{k, s-1} \Gamma$. This can be shown by making induction on the index $s . X_{0,0}$ can be written as $\widehat{X}_{0,0} \Gamma$ and we assume that it is true for all $X_{l, j-1}$ for $l=$ $0, \ldots, k$ and $j=1, \ldots, s-1$. Hence, $G_{k, s-1}$ can be written as $\widehat{G}_{k, s-1} \Gamma$, since $G_{k, s-1}=\sum_{l=0}^{k-1} \sum_{j=1}^{n_{l}} X_{l, j-1}+\sum_{j=1}^{s-1} X_{k, j-1}=$ $\left(\sum_{l=0}^{k-1} \sum_{j=1}^{n_{l}} \widehat{X}_{l, j-1}+\sum_{j=1}^{s-1} \widehat{X}_{k, j-1}\right) \Gamma$. Then (12) can be rewritten as

$$
\begin{aligned}
& X_{k, s-1}=\widehat{A}_{0} \Gamma+\sum_{j=1}^{N} A_{j} G_{k, s-1}^{j-1} \widehat{G}_{k, s-1} \Gamma-\widehat{G}_{k, s-1} \Gamma \\
& +\sum_{v=1}^{N} A_{v} G_{k}^{v-1} X_{k, s-1} \\
& +\sum_{j=1}^{N-1} \sum_{v=j+1}^{N} A_{v} G_{k}^{v-1-j} X_{k, s-1} G_{k}^{j-1} \widehat{G}_{k} \Gamma=(I \\
& \left.-\sum_{v=1}^{N} A_{v} G_{k}^{v-1}\right)^{-1} \times\left(\widehat{A}_{0}+\sum_{j=1}^{N} A_{j} G_{k, s-1}^{j-1} \widehat{G}_{k, s-1}\right. \\
& \left.-\widehat{G}_{k, s-1} \sum_{j=1}^{N-1} \sum_{v=j+1}^{N} A_{v} G_{k}^{v-1-j} X_{k, s-1} G_{k}^{j-1} \widehat{G}_{k}\right) \Gamma ;
\end{aligned}
$$

therefore $X_{k, s-1}$ can be decomposed as the product of an $m \times r$ matrix $\widehat{X}_{k, s-1}$ and an $r \times m$ matrix $\Gamma$. The inverse on the righthand side exists since $0 \leq \sum_{v=1}^{N} A_{v} G_{k}^{v-1} \leq \sum_{v=1}^{N} A_{v} G^{v-1}$ and the spectral radius of $\sum_{v=1}^{N} A_{v} G^{v-1}$ is strictly less than one [13]. Therefore we will concentrate on finding $\widehat{X}_{k, s-1}$ as the solution to

$$
\begin{aligned}
\widehat{X}_{k, s-1}= & \widehat{A}_{0}+\left(\sum_{j=1}^{N} A_{j} G_{k, s-1}^{j-1}-I\right) \widehat{G}_{k, s-1} \\
& +\sum_{v=1}^{N} A_{v} G_{k}^{v-1} \widehat{X}_{k, s-1} \\
& +\sum_{j=1}^{N-1} \sum_{v=j+1}^{N} A_{v} G_{k}^{v-1-j} \widehat{X}_{k, s-1} \Gamma G_{k}^{j-1} \widehat{G}_{k}
\end{aligned}
$$

$$
\begin{aligned}
= & \widehat{A}_{0}+\left(\sum_{j=1}^{N} A_{j} G_{k, s-1}^{j-1}-I\right) \widehat{G}_{k, s-1} \\
& +\sum_{j=0}^{N-1} S_{k, j+1} \widehat{X}_{k, s-1}\left(\Gamma \widehat{G}_{k}\right)^{j},
\end{aligned}
$$

which can be rewritten as

$$
\begin{gathered}
\left(S_{k, 1}-I\right) \widehat{X}_{k, s-1}+\sum_{j=1}^{N-1} S_{k, j+1} \widehat{X}_{k, s-1}\left(\Gamma \widehat{G}_{k}\right)^{j} \\
=\left(I-\sum_{j=1}^{N} A_{j} G_{k, s-1}^{j-1}\right) \widehat{G}_{k, s-1}-\widehat{A}_{0} .
\end{gathered}
$$

We can use the Schur decomposition method in $[11,12]$ to solve the above equation. Different from Newton's iteration in [11], the special coefficient structure can be reused here, thus saving the overall computational cost. We will report the numerical performance of the Newton-Shamanskii iteration in Section 6.

\section{The Case of Low-Rank Local and Upward Transitions}

In this section, the case of low-rank local and upward transitions is considered, where the $m \times m$ matrices $\left\{A_{i}, \quad 1 \leq i \leq N\right\}$ can be decomposed as $A_{i}=\Gamma \widehat{A}_{i}$ with $\Gamma \in \mathbb{R}^{m \times r}$ and $\widehat{A}_{i} \epsilon$ $\mathbb{R}^{r \times m}$. To exploit low-rank local and upward transitions, we introduce the matrix $U$, which is the generator of the censored Markov chain on level $i$, starting from level $i$, before the first transition on level $i-1$. The following equality holds based on a level crossing argument:

$$
U=\sum_{i=1}^{N} A_{i} G^{i-1}=\sum_{i=1}^{N} A_{i}\left((I-U)^{-1} A_{0}\right)^{i-1}
$$

For the case of low-rank local and upward transitions, we can rewrite $U$ as

$$
\begin{aligned}
U & =\sum_{i=1}^{N} A_{i}\left((I-U)^{-1} A_{0}\right)^{i-1} \\
& =\Gamma\left[\sum_{i=1}^{N} \widehat{A}_{i}\left((I-U)^{-1} A_{0}\right)^{i-1}\right]=\Gamma \widehat{U},
\end{aligned}
$$

which means that $U$ is of rank $r$, while $G=(I-U)^{-1} A_{0}$ is generally of rank $m$.

Therefore, we find $U$ as the solution to

$$
\mathscr{F}(X)=X-\sum_{i=1}^{N} A_{i}\left((I-X)^{-1} A_{0}\right)^{i-1}=0
$$

and get $G$ from $G=(I-U)^{-1} A_{0}[11,14]$. The NewtonShamanskii iteration step for (19) is as follows. 
For $k=0,1, \ldots$,

$$
\begin{aligned}
\mathscr{F}_{U_{k}}^{\prime} Y_{k, s-1} & =-\mathscr{F}\left(U_{k, s-1}\right), \\
U_{k, s} & =U_{k, s-1}+Y_{k, s-1}, \\
\qquad s=1,2, \ldots, n_{k}, & \\
U_{k+1} & =U_{k+1,0}=U_{k, n_{k}} .
\end{aligned}
$$

$Y_{k, s-1}$ is the solution to

$$
\begin{gathered}
Y_{k, s-1}-\sum_{i=2}^{N} A_{i} \sum_{j=1}^{i-1}\left(\left(I-U_{k}\right)^{-1} A_{0}\right)^{j-1}\left(I-U_{k}\right)^{-1} \\
\cdot Y_{k, s-1}\left(\left(I-U_{k}\right)^{-1} A_{0}\right)^{i-j}=\sum_{i=1}^{N} A_{i} \\
\cdot\left(\left(I-U_{k, s-1}\right)^{-1} A_{0}\right)^{i-1}-U_{k, s-1} .
\end{gathered}
$$

If we define $R_{k, j}=\sum_{i=j+1}^{N} A_{i}\left(\left(I-U_{k}\right)^{-1} A_{0}\right)^{i-1-j}\left(I-U_{k}\right)^{-1}$ and rearrange the terms, (21) can be rewritten as

$$
\begin{aligned}
Y_{k, s-1} & -\sum_{j=1}^{N-1} R_{k, j} Y_{k, s-1}\left(\left(I-U_{k}\right)^{-1} A_{0}\right)^{j} \\
= & \sum_{i=1}^{N} A_{i}\left(\left(I-U_{k, s-1}\right)^{-1} A_{0}\right)^{i-1}-U_{k, s-1},
\end{aligned}
$$

which is of the form $\sum_{j=0}^{N-1} B_{j} X C^{j}=E$. This iteration enables us to exploit low-rank local and upward transitions. The iterates $U_{k, s}=U_{k, s-1}+Y_{k, s-1}$, where $Y_{k, s-1}$ solves (21), can be rewritten as $U_{k, s}=\Gamma \widehat{U}_{k, s}$. This can be shown by making induction on the index $s$. It obviously holds for $U_{0,0}$. Assuming that $U_{k, s-1}=\Gamma \widehat{U}_{k, s-1}$, from (21), we get

$$
\begin{gathered}
Y_{k, s-1}=\Gamma\left[\sum_{i=2}^{N} \widehat{A}_{i} \sum_{j=1}^{i-1}\left(\left(I-U_{k}\right)^{-1} A_{0}\right)^{j-1}\left(I-U_{k}\right)^{-1}\right. \\
\cdot Y_{k, s-1}\left(\left(I-U_{k}\right)^{-1} A_{0}\right)^{i-j}+\sum_{i=1}^{N} \widehat{A}_{i} \\
\left.\cdot\left(\left(I-U_{k, s-1}\right)^{-1} A_{0}\right)^{i-1}-\widehat{U}_{k, s-1}\right]
\end{gathered}
$$

which tells us that $Y_{k, s-1}$ can be decomposed as $\Gamma \widehat{Y}_{k, s-1}$, and the same holds for $U_{k, s}=U_{k, s-1}+Y_{k, s-1}$. Therefore, from (21), we will focus on finding $\widehat{Y}_{k, s-1}$ as the solution to

$$
\begin{gathered}
\widehat{Y}_{k, s-1}-\sum_{i=2}^{N} \widehat{A}_{i} \sum_{j=1}^{i-1}\left(\left(I-U_{k}\right)^{-1} A_{0}\right)^{j-1}\left(I-U_{k}\right)^{-1} \\
\cdot Y_{k, s-1}\left(\left(I-U_{k}\right)^{-1} A_{0}\right)^{i-j}=\sum_{i=1}^{N} \widehat{A}_{i} \\
\cdot\left(\left(I-U_{k, s-1}\right)^{-1} A_{0}\right)^{i-1}-\widehat{U}_{k, s-1} .
\end{gathered}
$$

Defining $\widehat{R}_{k, j}=\sum_{i=j+1}^{N} \widehat{A}_{i}\left(\left(I-U_{k}\right)^{-1} A_{0}\right)^{i-1-j}\left(I-U_{k}\right)^{-1} \Gamma$, we can rewrite the above equation as

$$
\begin{aligned}
\widehat{Y}_{k, s-1} & -\sum_{j=1}^{N-1} \widehat{R}_{k, j} \widehat{Y}_{k, s-1}\left(\left(I-U_{k}\right)^{-1} A_{0}\right)^{j} \\
= & \sum_{i=1}^{N} \widehat{A}_{i}\left(\left(I-U_{k, s-1}\right)^{-1} A_{0}\right)^{i-1}-\widehat{U}_{k, s-1},
\end{aligned}
$$

which is of the form $\sum_{j=0}^{N-1} B_{j} X C^{j}=E$.

\section{Convergence Analysis}

There is monotone convergence when the Newton-Shamanskii method is applied to (3).

5.1. Preliminary. Let us first recall that a real square matrix $A$ is a $Z$-matrix if all its off-diagonal elements are nonpositive and can be written as $s I-B$ with $B \geq 0$. Moreover, a $Z$-matrix $A$ is called an $M$-matrix if $s \geq \rho(B)$, where $\rho(\cdot)$ is the spectral radius; it is a singular $M$-matrix if $s=\rho(B)$ and a nonsingular $M$-matrix if $s>\rho(B)$. The following result from [15] is to be exploited.

Lemma 1. For a Z-matrix $A$, the following statements are equivalent:
(a) $A$ is a nonsingular $M$-matrix.
(b) $A^{-1} \geq 0$.
(c) Av $>0$ for some vector $v>0$.
(d) All eigenvalues of A have positive real parts.

The following result is also well known [15].

Lemma 2. Let $A$ be a nonsingular $M$-matrix. If $B \geq A$ is a $Z$-matrix, then $B$ is a nonsingular $M$-matrix. Moreover, $B^{-1} \leq$ $A^{-1}$.

The minimal nonnegative solution $S$ for (3) may also be recalled-see [9] for details.

Theorem 3. If the rate $\rho$ defined by (5) satisfies $\rho \neq 1$, then the matrix

$$
I-\sum_{v=1}^{N} \sum_{j=0}^{v-1}\left(G^{v-1-j}\right)^{T} \bigotimes A_{v} G^{j}
$$

is a nonsingular M-matrix.

5.2. Monotone Convergence. The following lemma displays the monotone convergence properties of the Newton iteration for (3).

Lemma 4. Consider a matrix $X$ such that

(i) $\mathscr{G}(X) \geq 0$,

(ii) $0 \leq X \leq G$,

(iii) $I-\sum_{v=1}^{N} \sum_{j=0}^{v-1}\left(X^{v-1-j}\right)^{T} \otimes A_{v} X^{j}$ is a nonsingular $M$ matrix. 
Then the matrix

$$
Y=X-\left(\mathscr{G}_{X}^{\prime}\right)^{-1} \mathscr{G}(X)
$$

is well defined, and

(a) $\mathscr{G}(Y) \geq 0$,

(b) $0 \leq X \leq Y \leq G$,

(c) $I-\sum_{v=1}^{N} \sum_{j=0}^{v-1}\left(Y^{v-1-j}\right)^{T} \otimes A_{v} Y^{j}$ is a nonsingular $M-$ matrix.

Proof. $\mathscr{G}_{X}^{\prime}$ is invertible and the matrix $Y$ is well defined from (iii) and Lemma 1. Since

$$
\left[I-\sum_{v=1}^{N} \sum_{j=0}^{v-1}\left(X^{v-1-j}\right)^{T} \otimes A_{v} X^{j}\right]^{-1} \geq 0
$$

from (iii) and Lemma 1 and $\mathscr{G}(X) \geq 0$, we get that $\operatorname{vec}(Y) \geq$ $\operatorname{vec}(X)$ and thus $Y \geq X$. From (27) and the Taylor formula, there exists a number $\theta_{1}, 0<\theta_{1}<1$, such that

$$
\begin{aligned}
\mathscr{G}(Y)= & \mathscr{G}(X)+\mathscr{G}_{X}^{\prime}(Y-X) \\
& +\frac{1}{2} \mathscr{G}_{X}^{\prime \prime}\left(\theta_{1}(Y-X), \theta_{1}(Y-X)\right) \\
= & \frac{1}{2} \mathscr{G}_{X}^{\prime \prime}\left(\theta_{1}(Y-X), \theta_{1}(Y-X)\right) \geq 0,
\end{aligned}
$$

so (a) is proven, where the last inequality is obtained from $Y-X \geq 0$ and (8). (b) may be proven as follows. From

$$
\begin{aligned}
0= & \mathscr{G}(G) \\
= & \mathscr{G}(X)+\mathscr{G}_{X}^{\prime}(G-X) \\
& +\frac{1}{2} \mathscr{G}_{X}^{\prime \prime}\left(\theta_{2}(G-X), \theta_{2}(G-X)\right),
\end{aligned}
$$

where $0<\theta_{2}<1$, we have

$$
\begin{aligned}
-\mathscr{G}_{X}^{\prime}(G-Y) & =\mathscr{G}_{X}^{\prime}(Y-X)-\mathscr{G}_{X}^{\prime}(G-X) \\
& =-\mathscr{G}(X)-\mathscr{G}_{X}^{\prime}(G-X) \\
& =\frac{1}{2} \mathscr{G}_{X}^{\prime \prime}\left(\theta_{2}(G-X), \theta_{2}(G-X)\right) \geq 0,
\end{aligned}
$$

where the last inequality is from $G-X \geq 0$ by (ii). It is notable that

$$
I-\sum_{v=1}^{N} \sum_{j=0}^{v-1}\left(X^{v-1-j}\right)^{T} \bigotimes A_{v} X^{j}
$$

is a nonsingular $M$-matrix, $\operatorname{sovec}(G-Y) \geq 0$ from Lemma 1; that is, $G-Y \geq 0$. Now $Y \geq X$, so (b) follows. Next we prove (c). From $0 \leq Y \leq G$, we have

$$
\begin{aligned}
I & -\sum_{v=1}^{N} \sum_{j=0}^{v-1}\left(Y^{v-1-j}\right)^{T} \bigotimes A_{v} Y^{j} \\
& \geq I-\sum_{v=1}^{N} \sum_{j=0}^{v-1}\left(G^{v-1-j}\right)^{T} \bigotimes A_{v} G^{j},
\end{aligned}
$$

and we know that $I-\sum_{v=1}^{N} \sum_{j=0}^{v-1}\left(G^{v-1-j}\right)^{T} \otimes A_{v} G^{j}$ is a nonsingular $M$-matrix. Consequently, from Lemma 2, $I-$ $\sum_{v=1}^{N} \sum_{j=0}^{v-1}\left(Y^{v-1-j}\right)^{T} \otimes A_{v} Y^{j}$ is a nonsingular $M$-matrix.

A generalization of Lemma 4 provides the theoretical basis for the monotone convergence of the Newton-Shamanskii method for (3).

Lemma 5. Consider a matrix $X$ such that

(i) $\mathscr{G}(X) \geq 0$,

(ii) $0 \leq X \leq G$

(iii) $I-\sum_{v=1}^{N} \sum_{j=0}^{v-1}\left(X^{v-1-j}\right)^{T} \otimes A_{v} X^{j}$ is a nonsingular $M$ matrix.

Then, for any matrix $Z$, where $0 \leq Z \leq X$, the matrix

$$
Y=X-\left(\mathscr{G}_{Z}^{\prime}\right)^{-1} \mathscr{G}(X)
$$

exists, such that

(a) $\mathscr{G}(Y) \geq 0$

(b) $0 \leq X \leq Y \leq G$,

(c) $I-\sum_{v=1}^{N} \sum_{j=0}^{v-1}\left(Y^{v-1-j}\right)^{T} \otimes A_{v} Y^{j}$ is a nonsingular $M$ matrix.

Proof. Since $0 \leq Z \leq X$, we have

$$
\begin{aligned}
I- & \sum_{v=1}^{N} \sum_{j=0}^{v-1}\left(Z^{v-1-j}\right)^{T} \bigotimes A_{v} Z^{j} \\
& \geq I-\sum_{v=1}^{N} \sum_{j=0}^{v-1}\left(X^{v-1-j}\right)^{T} \bigotimes A_{v} X^{j} .
\end{aligned}
$$

From (iii) and Lemma $2, \mathscr{G}_{Z}^{\prime}$ is invertible and the matrix $Y$ is well defined such that $0 \leq X \leq Y$. Let

$$
\widehat{Y}=X-\left(\mathscr{G}_{X}^{\prime}\right)^{-1} \mathscr{G}(X)
$$

we know that $\widehat{Y} \geq Y$ from Lemma 2. Because $\widehat{Y} \leq G$ from Lemma 4, (b) follows. Now

$$
I-\sum_{v=1}^{N} \sum_{j=0}^{v-1}\left(\widehat{Y}^{v-1-j}\right)^{T} \bigotimes A_{v} \widehat{Y}^{j}
$$

is a nonsingular $M$-matrix from Lemma 4 and $\widehat{Y} \geq Y$; therefore $I-\sum_{v=1}^{N} \sum_{j=0}^{v-1}\left(Y^{v-1-j}\right)^{T} \otimes A_{v} Y^{j}$ is a nonsingular $M$ matrix from Lemma 2. Next we show that (a) is true. From 
the Taylor formula, there exists two numbers $\theta_{3}$ and $\theta_{4}$, where $0<\theta_{3}, \theta_{4}<1$, such that

$$
\begin{aligned}
\mathscr{G}(Y)= & \mathscr{G}(X)+\mathscr{G}_{X}^{\prime}(Y-X) \\
& +\frac{1}{2} \mathscr{G}_{X}^{\prime \prime}\left(\theta_{3}(Y-X), \theta_{3}(Y-X)\right) \\
= & \mathscr{G}(X)+\mathscr{G}_{Z}^{\prime}(Y-X)+\left(\mathscr{G}_{X}^{\prime}-\mathscr{G}_{Z}^{\prime}\right)(Y-X) \\
& +\frac{1}{2} \mathscr{G}_{X}^{\prime \prime}\left(\theta_{3}(Y-X), \theta_{3}(Y-X)\right) \\
= & \mathscr{G}_{Z}^{\prime \prime}\left((Y-X), \theta_{4}(X-Z)\right) \\
& +\frac{1}{2} \mathscr{G}_{X}^{\prime \prime}\left(\theta_{3}(Y-X), \theta_{3}(Y-X)\right) \geq 0,
\end{aligned}
$$

where the latter inequality holds, since $X-Z \geq 0$ and $Y-X \geq$ 0 .

The monotone convergence result for the Newton-Shamanskii method applied to (3) follows.

Theorem 6. Suppose that a matrix $G_{0}$ is such that

(i) $\mathscr{G}\left(G_{0}\right) \geq 0$,

(ii) $0 \leq G_{0} \leq G$,

(iii) $I-\sum_{v=1}^{N} \sum_{j=0}^{v-1}\left(G_{0}^{v-1-j}\right)^{T} \otimes A_{v} G_{0}^{j}$ is a nonsingular $M$ matrix.

Then the Newton-Shamanskii algorithm (9)-(10) generates a sequence $\left\{G_{k}\right\}$ such that $G_{k} \leq G_{k+1} \leq G$ for all $k \geq 0$, and $\lim _{k \rightarrow \infty} G_{k}=G$.

Proof. The proof is by mathematical induction. From Lemma 5,

$$
\begin{gathered}
G_{0}=G_{0,0} \leq \cdots \leq G_{0, n_{0}}=G_{1} \leq G, \\
\mathscr{G}\left(G_{1}\right) \geq 0, \\
I-\sum_{v=1}^{N} \sum_{j=0}^{v-1}\left(G_{1}^{v-1-j}\right)^{T} \bigotimes A_{v} G_{1}^{j}
\end{gathered}
$$

is a nonsingular $M$-matrix. Assuming that

$$
\begin{aligned}
\mathscr{G}\left(G_{i}\right) & \geq 0 \\
G_{0} & =G_{0,0} \leq \cdots \leq G_{0, n_{0}}=G_{1} \leq \cdots \leq G_{i-1, n_{i-1}} \\
& =G_{i} \leq G
\end{aligned}
$$

and that $I-\sum_{v=1}^{N} \sum_{j=0}^{v-1}\left(G_{i}^{v-1-j}\right)^{T} \otimes A_{v} X_{i}^{j}$ is a nonsingular $M$ matrix, from Lemma 5,

$$
\begin{aligned}
\mathscr{G}\left(G_{i+1}\right) & \geq 0, \\
G_{i} & =G_{i, 0} \leq \cdots \leq G_{i, n_{i}}=G_{i+1} \leq G,
\end{aligned}
$$

and $I-\sum_{v=1}^{N} \sum_{j=0}^{v-1}\left(G_{i+1}^{v-1-j}\right)^{T} \otimes A_{v} G_{i+1}^{j}$ is a nonsingular $M$ matrix. By induction, the sequence $\left\{G_{k}\right\}$ is therefore monotonically increasing and bounded above by $G$ and so has a limit $G_{*}$ such that $G_{*} \leq G$. Letting $i \rightarrow \infty$ in $G_{i+1} \geq$ $G_{i, 1}=G_{i}-\left(\mathscr{G}_{G_{i}}^{\prime}\right)^{-1} \mathscr{G}\left(G_{i}\right) \geq 0$, it follows that $\mathscr{G}\left(G_{*}\right)=0$. Consequently, $G_{*}=G$ since $G_{*} \leq G$ and $G$ is the minimal nonnegative solution of (3).

\section{Numerical Experiments}

The Newton-Shamanskii iteration differs from Newton's method in that the evaluation of the Fréchet derivative is not done at every iteration step. So, while more iterations will be needed than for Newton's method, the overall cost of the modified Newton method could be less. Our numerical experiments confirm the feasibility of the NewtonShamanskii iteration for (6).

About how to choose the optimal scalars $n_{i}$ in the Newton-like algorithm (see (9) and (10)), now we have no theoretical results. This is a goal for our future research. In our extensive numerical experiments, we update the Fréchet derivative every two iteration steps. That is, for $i=0,1, \ldots$, we choose $n_{i}=2$ in the Newton-like algorithm (9).

The elapsed CPU time in seconds (denoted as "time") is used to measure the feasibility of our new method. In our numerical experiments, we use zero matrix as the initial iteration value and choose the following stopping criterion:

$$
\left\|G_{\text {new }}-G_{\text {old }}\right\|<1 e-14
$$

where $G_{\text {new }}$ and $G_{\text {old }}$ denote the iteration values of solution after and before one iteration step, respectively, and $\|\cdot\|$ denotes the infinity-norm of a matrix. The numerical tests were performed on a laptop $(2.4 \mathrm{GHz}$ and $2 \mathrm{G}$ Memory) with MATLAB R2013b. Numerical experiments show that the modified Newton method could be more efficient than Newton iteration proposed in [11]. We present the numerical results for a random-generated problem in Figure 1. In this figure, we fix the number of coefficient matrices $(N+1)$ and vary the problem size $(n)$ and plot the CPU time of the two algorithms in seconds for different parameters $n$.

The MATLAB code used for the problem construction is reported as follows, which generates the $N+1$ coefficient matrices for (6), that is, $A_{i} \in \mathbb{R}^{n \times n}, i=0,1,2, \ldots, N$ :

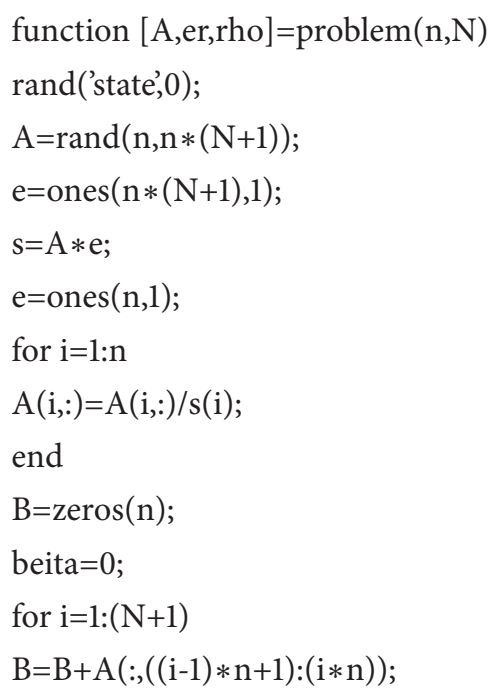



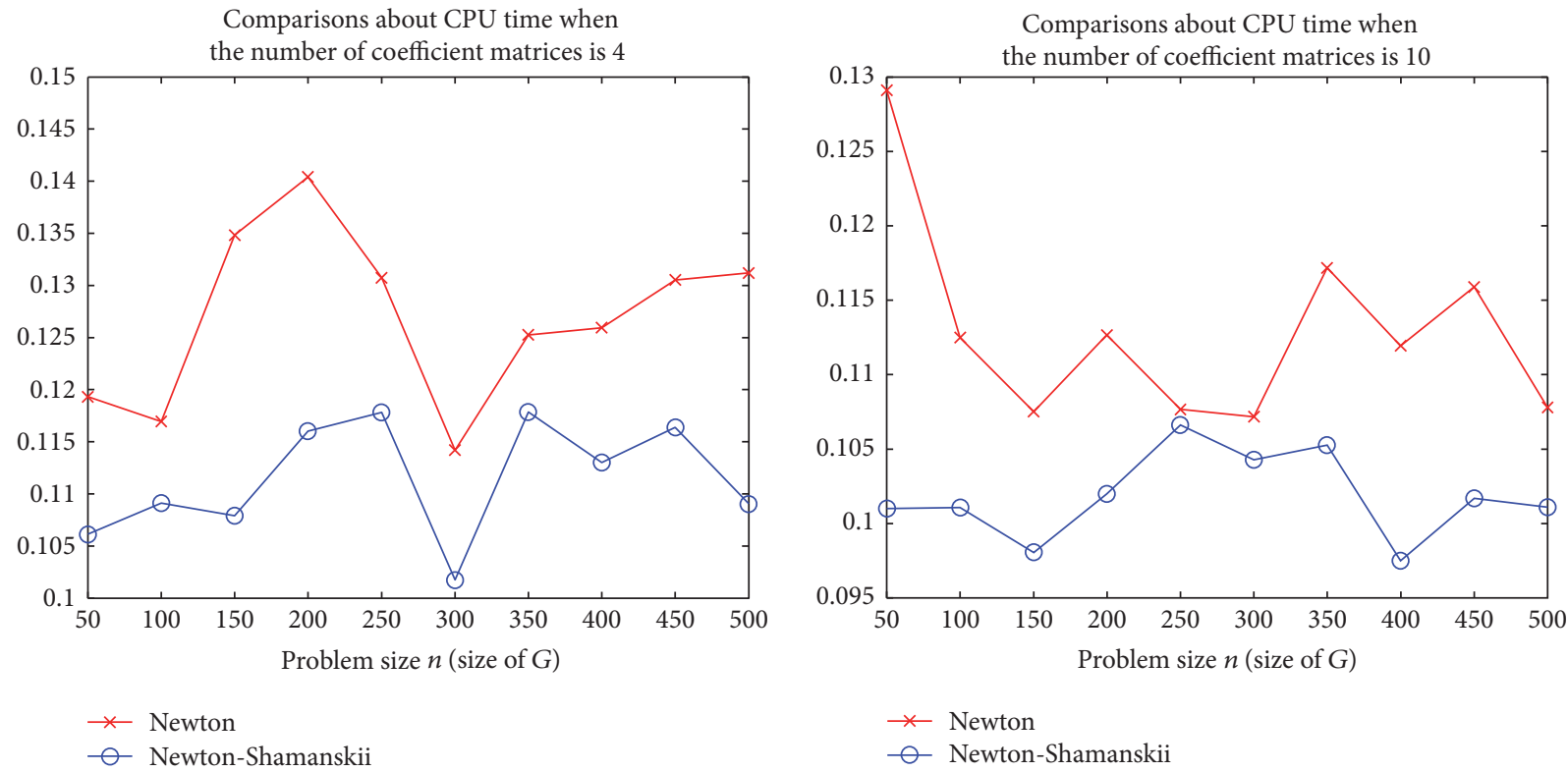

FIgURE 1: Comparisons about CPU time.

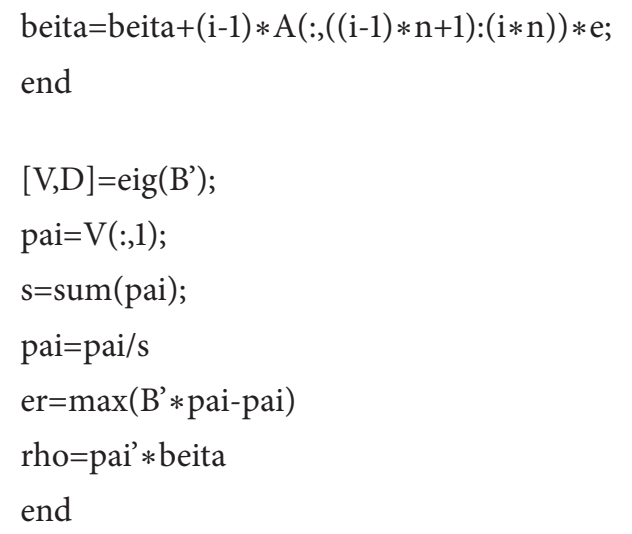

Up to now, we do not have theoretical results about when this modified Newton method can outperform the Newton iteration. This is not an easy question. We leave it as a goal for future research.

\section{Conflicts of Interest}

The author declares that there are no conflicts of interest regarding the publication of this paper.

\section{Acknowledgments}

This research is supported by the Fundamental Research Funds for the Central Universities in China University of Geosciences, Beijing (2652017140).

\section{References}

[1] M. F. Neuts, Structured Stochastic Matrices of the M/G/1 Types and Their Applications, vol. 5 of Probability: Pure and Applied, Marcel Dekker, New York, NY, USA, 1989.
[2] M. Neuts, Matrix-Geometric Solutions in Stochastic Models-An Algorithmic Approach, The Johns Hopkins University Press, Baltimore, Md, USA, 1981.

[3] V. Ramaswami, "Nonlinear matrix equations in applied probability - solution techniques and open problems," SIAM Review. A Publication of the Society for Industrial and Applied Mathematics, vol. 30, no. 2, pp. 256-263, 1988.

[4] V. Ramaswami, "A duality theorem for the matrix paradigms in queueing theory," Communications in Statistics. Stochastic Models, vol. 6, no. 1, pp. 151-161, 1990.

[5] P. G. Taylor and B. Van Houdt, "On the dual relationship between Markov chains of GI/M/1 and M/G/1 type," Advances in Applied Probability, vol. 42, no. 1, pp. 210-225, 2010.

[6] D. Bini and B. Meini, "On the solution of a nonlinear matrix equation arising in queueing problems," SIAM Journal on Matrix Analysis and Applications, vol. 17, no. 4, pp. 906-926, 1996.

[7] N. Akar and K. Sohraby, "An invariant subspace approach in M/G/1 and G/M/1 type Markov chains," Communications in Statistics. Stochastic Models, vol. 13, no. 3, pp. 381-416, 1997.

[8] D. Bini, B. Meini, and V. Ramaswami, "Analyzing M/G/1 paradigms through QBDs: the role of the block structure in computing the matrix G," in Advances in Algorithmic Methods for Stochastic Models, G. Latouche and P. Taylor, Eds., pp. 7386, Notable Publications: Neshanic, Station, NJ, 2000.

[9] G. Latouche, "Newton's iteration for non-linear equations in Markov chains," IMA Journal of Numerical Analysis (IMAJNA), vol. 14, no. 4, pp. 583-598, 1994.

[10] M. F. Neuts, "Moment formulas for the Markov renewal branching process," Advances in Applied Probability, vol. 8, no. 4, pp. 690-711, 1976.

[11] J. F. Pérez, M. Telek, and B. Van Houdt, "A Fast Newton's Iteration for M/G/1-Type and GI/M/1-Type Markov Chains," Stochastic Models, vol. 28, no. 4, pp. 557-583, 2012.

[12] J. F. Pérez and B. Van Houdt, "The M/G/1-type Markov chain with restricted transitions and its application to queues with 
batch arrivals," Probability in the Engineering and Informational Sciences, vol. 25, no. 4, pp. 487-517, 2011.

[13] D. A. Bini, G. Latouche, and B. Meini, Numerical methods for structured Markov chains, Numerical Mathematics and Scientific Computation, Oxford University Press, New York, 2005.

[14] G. Latouche and V. Ramaswami, Introduction to matrix analytic methods in stochastic modeling, ASASIAM Series on Statistics and Applied Probability; SIAM, Philadelphia, PA, USA, 1999.

[15] R. S. Varga, Matrix Iterative Analysis, Prentice Hall, Englewood Cliffs, NJ, USA, 1962. 


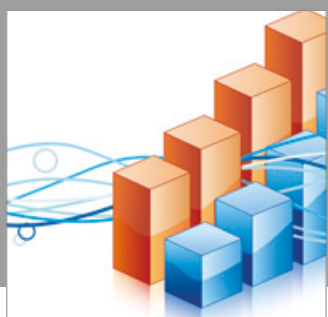

Advances in

Operations Research

vatersals

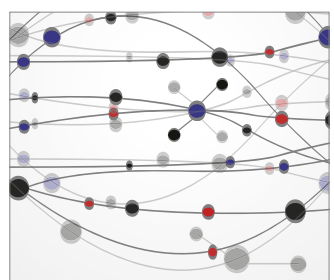

\section{The Scientific} World Journal
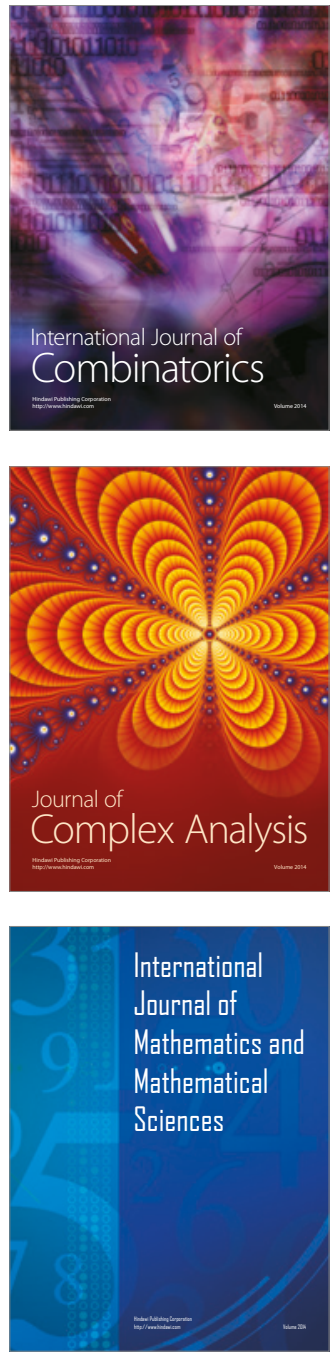
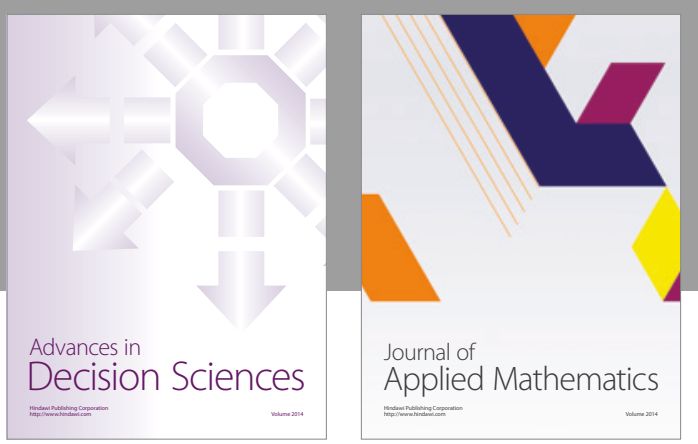

Algebra

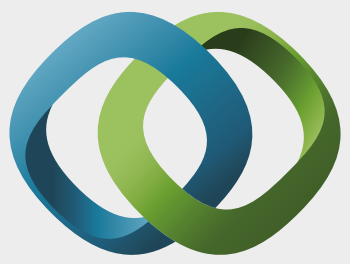

\section{Hindawi}

Submit your manuscripts at

https://www.hindawi.com
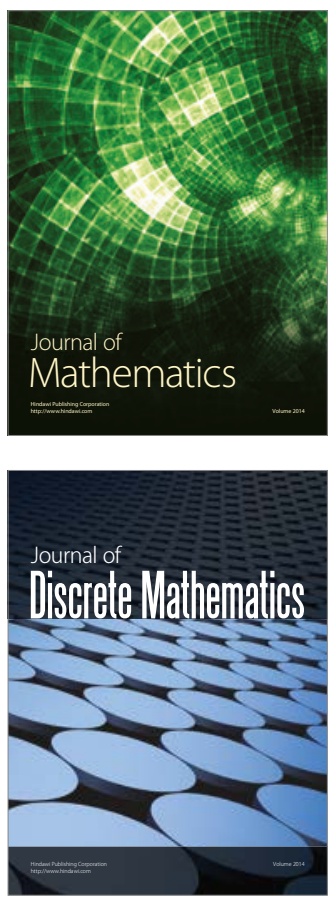

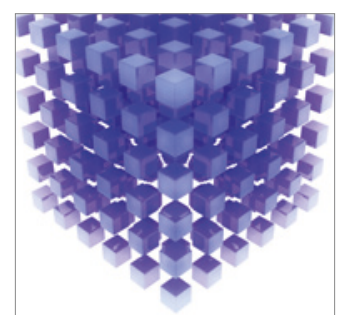

Mathematical Problems in Engineering
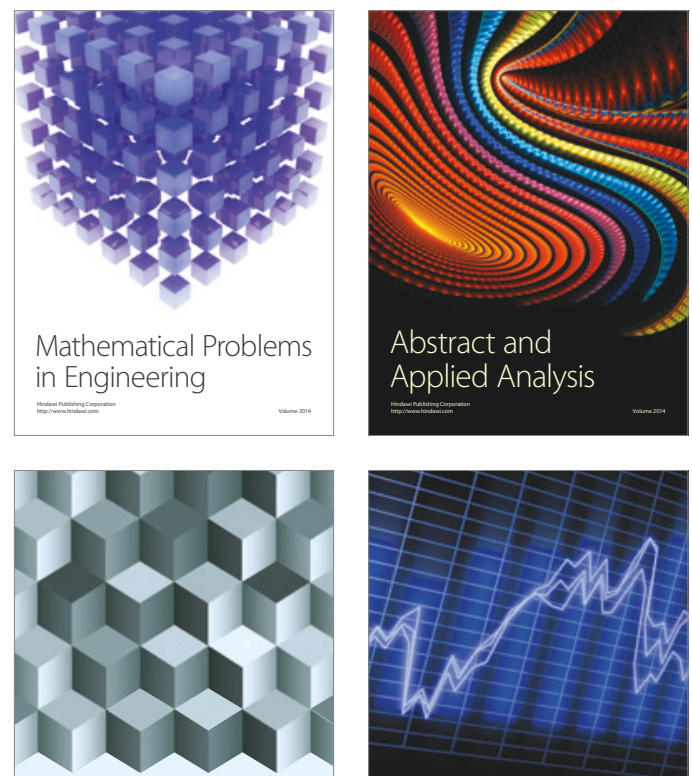

Journal of

Function Spaces

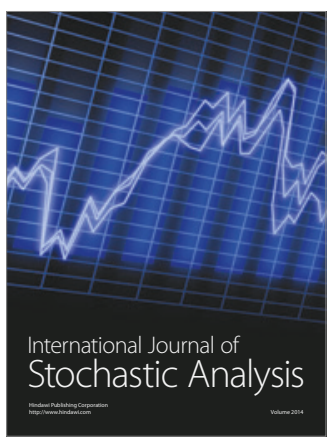

Probability and Statistics
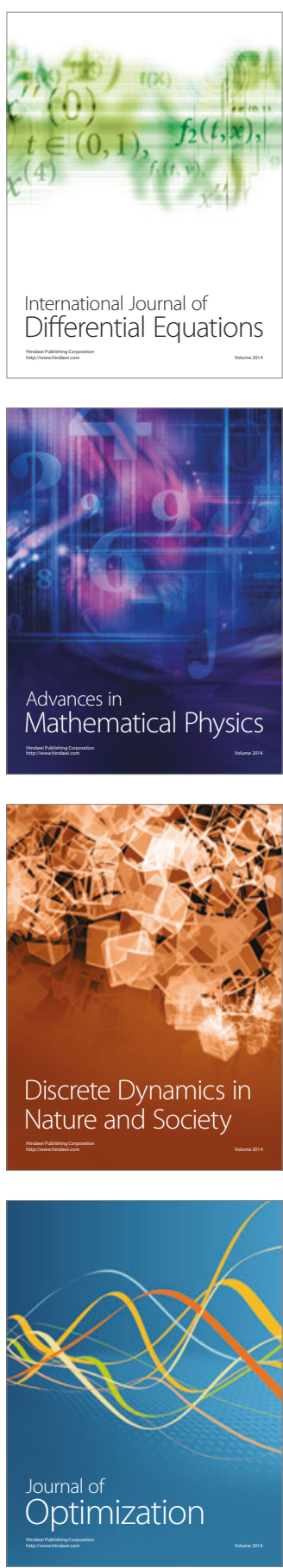